\title{
Furcotanilla, a New Genus of the Ant Subfamily Leptanillinae from China with Descriptions of Two New Species of Protanilla and $P$. rafflesi Taylor (Hymenoptera: Formicidae)
}

by

\author{
Zheng-Hui $\mathrm{Xu}^{1}$
}

\section{ABSTRACT}

A new genus of the ant subfamily Leptanillinae, Furcotanilla gen. nov., discovered in southwestern China is described. The new genus is distributed in the Oriental region and belongs to the tribe Anomalomyrmini of Leptanillinae. A key to the 4 known genera of Leptanillinae of the world based on the worker caste is provided. Two new species of Protanilla collected from southwestern China, P. gengma sp. nov. and P. tibeta sp. nov., are described. The type-species of Protanilla, P. rafflesi Taylor, is also described based on the AntWeb images. A key to the 7 known species of Protanilla of the world based on worker and queen castes is prepared.

Key words: Hymenoptera, Formicidae, Leptanillinae, Furcotanilla, New genus, New species.

\section{INTRODUCTION}

After the establishment of Protanilla Taylor and Anomalomyrma Taylor (Bolton, 1990, 1994), 6 new species of Protanilla were described in the world (Bolton, 1995, 2011; Xu, 2002; Xu \& Zhang, 2002; Baroni Urbani \& De Andrade, 2006; Terayama, 2009). But the worker caste of Anomalomyrma remained unknown. Recently, Borowiec et al. (2011) described 2 new species of Anomalomyrma from the Phillipines and Malaysia, and the first discovery of the worker caste has provided sufficient knowledge for the identification of Anomalomyrma.

A new genus, Furcotanilla gen. nov., based on the type species Protanilla furcomandibula Xu et Zhang (2002) from southwestern China is erected. The new genus is distributed in the Oriental region and belongs to the tribe

\footnotetext{
${ }^{1}$ Key Laboratory of Forest Disaster Warning and Control in Yunnan Province, College of Forestry, Southwest Forestry University, Kunming, Yunnan Province 650224, China E-mail: xuzhenghui1962@163.com
} 
Anomalomyrmini of Leptanillinae. In order to understand the differentiation between the new genus and other ones, a key to the 4 known genera of Leptanillinae of the world based on the worker caste is provided.

Two new species of Protanilla collected from southwestern China, P.gengma sp. nov. and $P$. tibeta sp. nov., are described. The type species of Protanilla, $P$. rafflesi Taylor, is also described based on the AntWeb images. A key to the 7 known species of Protanilla of the world based on worker and queen castes is prepared.

\section{MATERIALS AND METHODS}

The worker caste of Protanilla gengma sp. nov. and P. tibeta sp. nov. were collected by the sample-plot method. Descriptions and measurements were made under a XTB- 1 stereo microscope with a micrometer. Illustrations were made under a Motic-700Z stereo microscope with illustrative equipment.

Standard measurements and indices are as defined in Bolton (1987) and Xu \& Zhang (2002):

TL-Total Length: The total outstretched length of the ant from the mandibular apex to the gastral apex.

HL-Head Length: The length of the head proper, excluding the mandibles, measured in a straight line from the mid-point of the anterior clypeal margin to the mid-point of the occipital margin, in full-face view. In species where the occipital margin or the clypeal margin is concave, the measurement is taken from the mid-point of a transverse line spanning the anteriormost or posteriormost projecting points, respectively.

HW-Head Width: The maximum width of the head in full face view, excluding the eyes.

CI-Cephalic Index $=\mathrm{HW} \times 100 / \mathrm{HL}$.

SL-Scape Length: The maximum straight line length of the antennal scape excluding the basal constriction or neck close to the condylar bulb.

SI-Scape Index $=$ SL $\times 100 / \mathrm{HW}$.

ML-Mandibular length: The straight-line length of the mandible from apex to the base.

PW-Pronotal Width: The maximum width of the pronotum in dorsal view.

AL-Alitrunk Length: The diagonal length of the alitrunk in profile view 
from the point at which the pronotum meets the cervical shield to the posterior base of the metapleuron.

PNL-Petiolar node length: With petiolar node in lateral view, the maximum longitudinal length of the node without its anterior and posterior peduncles.

PNH-Petiolar node height: With petiolar node in lateral view, the maximum vertical height of the node from summit to lowermost part of subpetiolar process.

PNW-Petiolar node width: The maximum width of the petiolar node in dorsal view.

PPNL-Postpetiolar node length: With postpetiolar node in lateral view, the maximum longitudinal length of the node without its anterior and posterior peduncles.

PPNH-Postpetiolar node height: With postpetiolar node in lateral view, the maximum vertical height of the node from summit to lowermost part of subpostpetiolar process.

PPNW-Postpetiolar node width: The maximum width of the postpetiolar node in dorsal view.

The measurements of ML, PNL, PNH, PNW, PPNL, PPNH, and PPNW of Protanilla rafflesi Taylor are obtained from the AntWeb images according to the scale bar.

All measurements are expressed in millimeters.

The type specimens are deposited in the Insect Collection, Southwest Forestry University (SWFU), Kunming, Yunnan Province, China.

\section{KEY TO KNOWN GENERA OF LEPTANILLINAE OF THE WORLD BASED ON WORKER CASTE}

1. Mandibles subtriangular, masticatory margins shorter than or subequal to inner margins, the former with 3-5 teeth. Antennal insertions very close to the anterior margin of the head. Body very slender (Old World Tropics and Temperate Regions) (Figs. 1-4) ......................... Leptanilla Emery, 1870

- Mandibles elongate, masticatory margins much longer than inner margins, the former with many more than 5 minute saw-like denticles or slender peg-like teeth. Antennal insertions well behind the anterior margin of the head. Body relatively robust. 


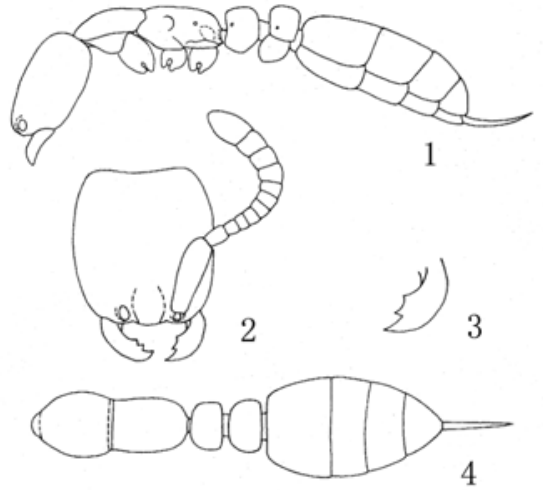

Figs. 1-4: Worker of Leptanilla yunnanensis Xu; 1. Head and body in profile view; 2 . Head in full face view; 3. Mandible in dorsal view; 4. Body in dorsal view. (Cited from Xu, 2002. Pilosity omitted).

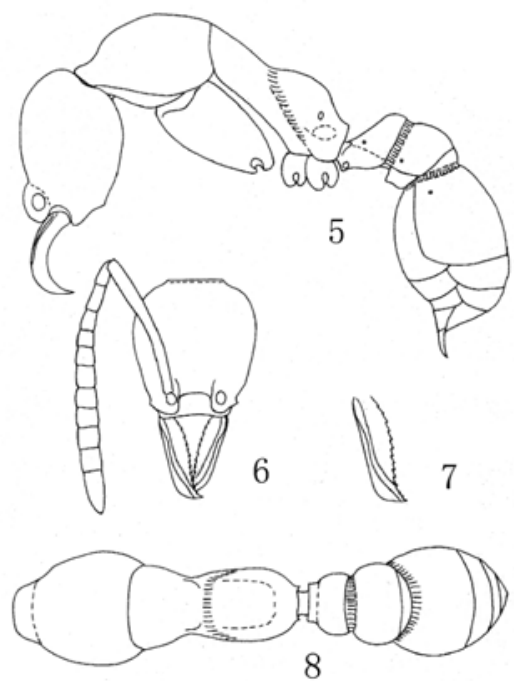

Figs. 5-8: Worker of Anomalomyrma boltoni Borowiec et al.; 5. Head and body in profile view; 6. Head in full face view; 7. Mandible in dorsal view; 8. Body in dorsal view. (Drawn from images of Borowiec et al., 2011. Pilosity omitted).
2. Masticatory mrgins of mandibles with many minute saw-like denticles. In profile view, promesonotum high and strongly convex. Metanotal groove deeply depressed. Petiole broadly attached to the postpetiole (Indo-Australian) (Figs. 5-8) Anomalomyrma Taylor, 1990 - Masticatory margins of mandibles with many slender peg-like teeth. In profileview, promesonotum low and weakly convex. Metanotal groove shallowly notched. Petiole narrowly attached to the postpetiole............ 3 3. In profile view, mandibles massive, lateroventral margin furcated and with 2 teeth. Postpetiole broadly attached to anterior face of gaster. Ventral face of postpetiole concave. In dorsal view, anterior margin of gaster deeply concave (Oriental) (Figs. 9-12)..Furcotanilla gen. nov. - In profile view, mandibles slender, lateroventral margin not furcated and without teeth. Postpetiole narrowly attached to anterior face of gaster. Ventral face of postpetiole convex. In dorsal view, anterior margin of gaster almost straight (Southern Palaearctic, Oriental, and Indo-Australian) (Figs. 13-24) ...... Protanilla Taylor, 1990. 


\section{DESCRIPTION OF NEW GENUS}

\section{Furcotanilla gen. nov.}

(Figs. 9-12)

\section{Type-species: Protanilla furcomandibula Xu et Zhang}

\section{Gender: Feminine.}

Etymology: The name of the new genus is a combination of "furco-", descriptive of the furcated mandibles found in the type-species, and the root "-tanilla", commonly used in Leptanillinae.

Diagnosis of worker: Robust terrestrial leptanilline ants with the following combination of characters.

Head elongate and narrowed forward, anterolateral corners toothed, strongly constricted before the teeth.

Mandibles massive, elongate-triangular and strongly down-curved apically.

Lateroventral margin of mandible with a long tooth and a short tooth, look-like furcated.

Laterodorsal surface of mandible with a longitudinal groove.

Masticatory margin of mandible with numerous slender peg-like teeth, but crenulated at the down-curved apical third.

Apex of mandible with a long stout hair on the ventral face.

Clypeus trapezoidal, widened forward, anterior margin concave.

Antennal insertions well behind the anterior margin of head.

Antennaelong, 12-segmented, scapes distinctly surpassed occipital corners, flagella filiform.

Eyes absent.

Mesonotum strongly constricted.

Promesonotal suture present.

Metanotal groove shallowly depressed.

Propodeal spiracles small and circular, lower-down on the side.

Metapleural bullae elongate elliptical, horizontal and close to the spiracles.

Petiole narrowly attached to postpetiole, petiolar node roughly square, subpetiolar process protruding, with large nearly circular semitransparent fenestra. 
Postpetiole broadly attached to the anterior face of gaster, ventral face of postpetiole deeply concave.

Lateral sides of the first gastral segment deeply and narrowly notched between the tergite and sternite at the anterior margin.

In dorsal view, anterior margin of gaster deeply concave in order to accept the laterally compressed postpetiole.

Sting strong and extruding.

Cuticle smooth and shining.

Pilosity sparse.

Female and male: Unknown.

Comparison: The new genus is close to Protanilla Taylor (Figs. 13-24), but with the mandibles massive and furcated on the lateroventral margin; postpetiole broadly attached to the anterior face of gaster, ventral face of postpetiole deeply concave; anterior margin of gaster deeply concave in order to accept the postpetiole.

Systematic position: Leptanillinae: Anomalomyrmini.

Geographical range: Oriental.

Discussion: Xu \& Zhang(2002) described an interesting species Protanilla furcomandibula from Yunnan, China. It is a very special species with furcated mandibles, ventrally concaved postpetiole, broad articulation between postpetiole and gaster, and anteriorly concaved gaster, all these characters do not exist in the other species of Protanilla.After a re-observation of the species, I realized that $P$. furcomandibula represented a new genus of Leptanillinae, Furcotanilla gen. nov., which has distinctly divided from Protanilla. The type species, P. furcomandibula, is therefore transferred from Protanilla to Fur-

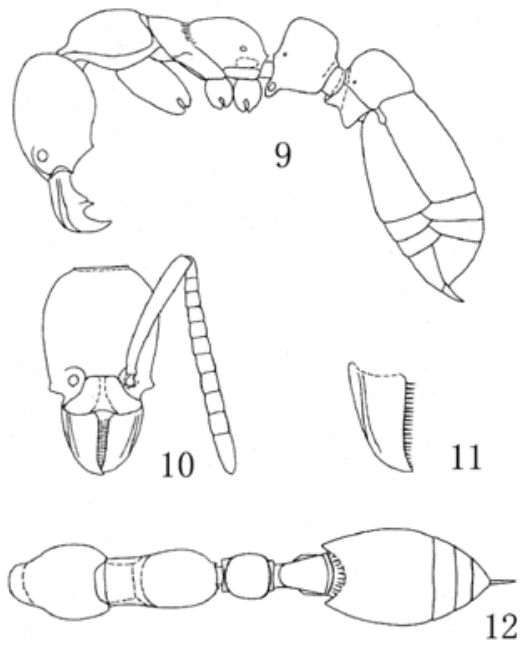

Figs. 9-12: Worker of Furcotanilla furcomandibula (Xu etZhang); 9. Head and body in profile view; 10. Head in full face view; 11. Mandible in dorsal view; 12. Body in dorsal view. (Cited from Xu \& Zhang, 2002, slightly modified. Pilosity omitted). 
cotanilla. The new genus is distributed in the Oriental region and belongs to the tribe Anomalomyrmini of Leptanillinae based on the elongate head, elongate mandibles, trapezoidal clypeus, well behind antennal insertions, elongate antennae, depressed metanotal groove, developed subpetiolar process, and relatively robust body.

\section{TRANSFERENCE OF SPECIES}

\section{Furcotanilla furcomandibula (Xu et Zhang) com. nov.}

(Figs. 9-12)

Protanilla furcomandibula Xu et Zhang, 2002: 140-142, figs. 1-3. Holotype worker and paratype worker, CHINA: Yunnan Province, Kunming, Xishan Forest Park, Huatingsi Temple, $2250 \mathrm{~m}$, collected in a soil sample in conifer-broadleaf mixed forest, 2001.III.31, Zheng-Hui Xu leg., No.A00250. [Holotype worker and paratype worker in SWFU examined.]

Remarks: Currently, Furcotanilla furcomandibula (Xu et Zhang) is the only known species in the new genus. According to the collection information, the species nests in the soil and forages on the ground in conifer-broadleaf mixed forest at altitudes around $2250 \mathrm{~m}$.

\section{KEY TO KNOWN SPECIES OF PROTANILLA OF THE WORLD BASED ON WORKER AND QUEEN CASTES}

1. In full face view, anterior margin of clypeus strongly concave. Laterodorsal surface of mandible without longitudinal groove. In dorsal view, petiolar node laterally compressed and roughly elliptic, distinctly longer than broad. Body bicolor, the middle portion black, the rest brownish yellow............2

- In full face view, anterior margin of clypeus straight to weakly concave. Laterodorsal surface of mandible with a longitudinal groove. In dorsal view, petiolar node anteroposteriorly compressed or square, as broad as long or broader than long. Body concolor, uniformly yellowish brown or reddish brown ................................................................................................ 3

2. In full face view, anterior $1 / 3$ of the head distinctly narrowed forward. In profile view, anterodorsal corner of petiolar node roundly prominent. Head brownish yellow. Body small with TL 2.7-3.0 mm (Xu, 2002: figs. 21-23) (China: Yunnan Province).... P. bicolor $\mathrm{Xu}$ 
- In full face view, anterior $1 / 2$ of the head distinctly narrowed forward. In profile view, anterodorsal corner of petiolar node rounded. Head light black to blackish brown. Body large with TL 4.1-4.5 mm (Figs. 13-16) (China: Yunnan Province)

P. gengma sp. nov.

3. In dorsal view, petiolar node nearly square, as broad as long, and weakly narrowed backward. In profile view, anterior face of petiolar node vertical, postpetiolar node not inclined forward (Terayama, 2009: figs. 113-118) (China: Taiwan Province).

P. lini Terayama

- In dorsal view, petiolar node anteroposteriorly compressed, distinctly broader than long, and weakly widened backward. In profile view, anterior face of petiolar node steeply sloped. Postpetiolar node strongly inclined forward

4. In full face view, occipital margin almost straight. In dorsal view, anterior margin of gaster weakly concave (Baroni Urbani \& De Andrade, 2006: figs. 1-3) (Sri Lanka)................... P. schoedli Baroni Urbani et De Andrade

- In full face view, occipital margin weakly concave. In dorsal view, anterior margin of gaster straight .5

5. In full face view, anterior $1 / 2$ of the head distinctly narrowed forward. In profile view, petiolar node relatively thin and roughly triangular, dorsal face very short, about 1/2 length of anterior face (Xu, 2002: figs. 18-20) (China: Yunnan Province) P. concolor $\mathrm{Xu}$

- In full face view, anterior $1 / 3$ of the head distinctly narrowed forward. In profile view, petiolar node relatively thick and roughly trapezoidal, dorsal face long, about as long as anterior face.

6. In profile view, posterodorsal corner of propodeum evenly convex. Petiolar node strongly narrowed upward, anterior face nearly straight, dorsal face weakly convex, anterodorsal corner blunt (Figs. 17-20) (China: Tibet)... P. tibeta sp. nov.

- In profile view, posterodorsal corner of propodeum strongly convex. Petiolar node weakly narrowed upward, anterior face weakly concave, dorsal face strongly convex, anterodorsal corner roundly protruding (Figs. 21-24) (Singapore, Malaysia) P. rafflesi Taylor 


\section{DESCRIPTIONS OF NEW SPECIES AND PROTANILLA} RAFFLESI

\section{Protanilla gengma sp. nov.}

(Figs. 13-16)

Holotype worker: TL 4.1, HL 0.73, HW 0.60, CI 83, SL 0.68, SI 113, ML 0.43, PW 0.48, AL 1.20, PNL 0.38, PNH 0.48, PNW 0.26, PPNL 0.35, PPNH 0.48, PPNW 0.28.

In full face view, head longer than broad, anterior $1 / 2$ of the head distinctly narrowed forward. Lateral sides evenly convex, anterolateral corners of head prominent and tooth-like. Occipital margin weakly concave, occipital corners rounded. Mandibles elongate and down-curved apically, dorsolateral surface without a longitudinal groove, basal corners roundly prominent, masticatory margin with about 21 peg-like teeth. Clypeus nearly trapezoidal, anterior margin evenly concave. Apex of labrum roundly convex, with 4 peg-like teeth. Antennae 12-segmented, apices of scapes surpassed occipital corners by about $1 / 6$ of its length, flagella segments $4-9$ about as broad as long.

In profile view, dorsum of prono-

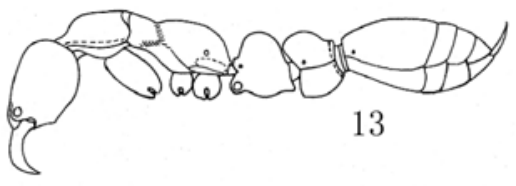
tum nearly straight. Promesonotal suture complete but not depressed. Dorsum of mesonotum straight, slope down backward. Metano-

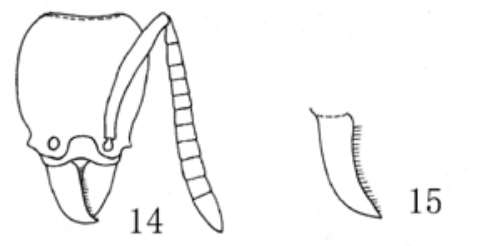
tal groove moderately notched. Dorsum of propodeum slightly convex, weakly slope down backward, posterodorsal corner evenly convex; declivity weakly convex, about $1 / 2$ length of the dorsum.

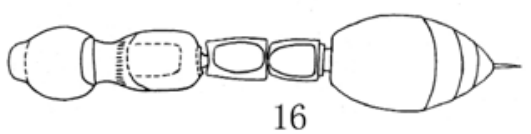
Petiolar node nearly trapezoidal, anterior and posterior faces nearly straight, dorsal face roundly con-

Figs. 13-16: Worker of Protanilla gengma sp. nov.; 13. Head and body in profile view; 14. Head in full face view; 15. Mandible in dorsal view; 16. Body in dorsal view. (Pilosity omitted). vex; anterodorsal corner slightly higher the posterodorsal corner, both are rounded. Ventral face of petiole roundly convex, anteroven- 
tral corner roundly extruding, with a circular semitransparent fenestra; posteroventral corner acutely angled. Postpetiolar node strongly inclined forward, with dorsum roundly convex, anterodorsal corner bluntly prominent, anterior face straight; ventral face roundly convex, anteroventral corner bluntly angled. Sting strong and extruding.

In dorsal view, mesonotum strongly constricted. Petiolar node nearly rectangular, longer than broad, length $:$ width $=1.4: 1$, lateral sides evenly convex, anterior face nearly straight, posterior face roundly convex. Postpetiolar node trapezoidal and widened backward, longer than broad, length : width $=1.3: 1$; lateral sides weakly convex, anterior face roundly convex, posterior face nearly straight. Anterior margin of gaster straight.

Mandibles sparsely finely punctured. Head and body smooth and shining. Dorsa of head and gaster with abundant erect to suberect hairs and dense decumbent pubescence. Alitrunk, petiole, and postpetiole with sparse erect to suberect hairs and abundant decumbent pubescence. Scapes and tibiae with abundant suberect to subdecumbent hairs and dense decumbent pubescence. Mouthparts with abundant longer hairs, apex of mandible with a stout long hair. Head light black. Mandibles, antennae, prothorax, legs, and gastral segments 2-4 yellowish brown. Mesothorax, metathorax, propodeum, petiole, postpetiole, and first gastral segment black.

Paratype workers: TL 4.1-4.5, HL 0.70-0.78, HW 0.60-0.65, CI 83-87, SL 0.65-0.73, SI 104-113, ML 0.43-0.48, PW 0.48-0.51, AL 1.18-1.30, PNL 0.35-0.38, PNH 0.48-0.50, PNW 0.26-0.29, PPNL 0.33-0.35, PPNH 0.480.51, PPNW 0.28-0.30 (6 individuals measured.). As holotype, but head light black to blackish brown.

Holotype: worker, CHINA:Yunnan Province, Gengma County, Mengding Town, Nantianmen, $1760 \mathrm{~m}$, collected from a soil sample in monsoon evergreen broadleaf forest, 2011.III.10, Hai-Bin Li leg., No. A11-29.

Paratypes: 6 workers, with the same data as holotype.

Comparison: This new species is close to P. bicolor $\mathrm{Xu}$, but in full face view, anterior $1 / 2$ of the head distinctly narrowed forward; in profile view, anterodorsal corner of petiolar node rounded; head light black to blackish brown; body large with TL 4.1-4.5 mm.

Etymology: The new species is named after the type locality "Gengma". 


\section{Protanilla tibeta sp. nov.}

(Figs. 17-20)

Holotype worker: TL 2.6, HL 0.53, HW 0.40, CI 76, SL 0.43, SI 106, ML 0.28, PW 0.33, AL 0.75, PNL 0.18, PNH 0.30, PNW 0.23, PPNL 0.20, PPNH 0.30, PPNW 0.23.

In full face view, head longer than broad, anterior $1 / 3$ of the head distinctly narrowed forward. Lateral sides weakly convex, anterolateral corners prominent and tooth-like, strongly concave before the teeth. Occipital margin weakly concave. Occipital corners roundly prominent. Mandibles elongate and down-curved apically, laterodorsal surface with a longitudinal groove, basal corners roundly prominent, masticatory margin with about 12 peg-like teeth. Clypeus roughly trapezoidal, anterior margin nearly straight. Antennae 12-segmented, apices of scapes just reached to occipital corners, flagella segments 2-10 about as broad as long.

In profile view, pronotum weakly convex. Promesonotal suture complete but not depressed. Dorsum of mesonotum straight and weakly slope down backward. Metanotal groove shallowly depressed. Dorsum of propodeum

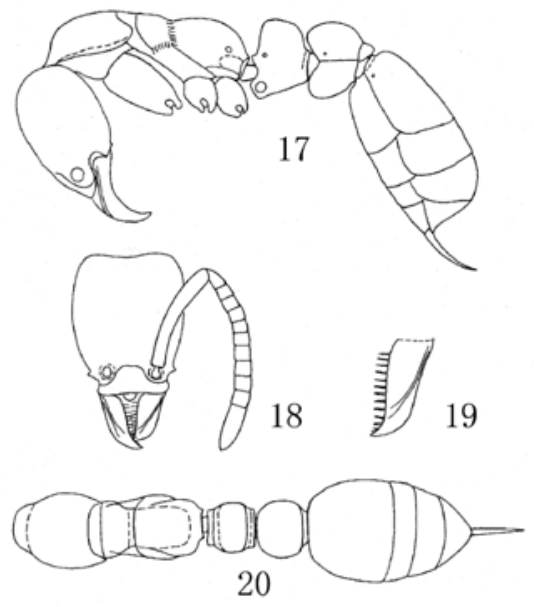

Figs. 17-20: Worker of Protanilla tibeta sp. nov.; 17. Head and body in profile view; 18 . Head in full face view; 19. Mandible in dorsal view; 20. Body in dorsal view. (Pilosity omitted). weakly convex, posterodorsal corner evenly convex; declivity weakly convex, about $1 / 2$ length of the dorsum. Propodeal spiracle circular and small, lower down on the side. Metapleural bulla elongate and roughly elliptical, close to the spiracle. Petiolar node nearly trapezoidal, anterior face nearly straight, about as long as dorsal face, dorsal and posterior faces weakly convex; anterodorsal corner blunt, higher than posterodorsal corner, the latter rounded; ventral face oblique and straight, anteroventral process roughly square, with circular semitransparent fenestra. Postpetiolar 
node strongly inclined forward, dorsum roundly convex, anterodorsal corner bluntly prominent, anterior face straight; ventral face roundly convex, anteroventral corner roundly protruding. Sting strong and extruding.

In dorsal view, petiolar node broader than long, width : length $=1.4: 1$, slightly widened backward; lateral sides weakly convex, anterior and posterior faces nearly straight. Postpetiolar node about as broad as long, width: length $=$ 1.1:1, weakly widened backward, lateral sides and anterior face roundly convex, posterior face nearly straight. Anterior margin of gaster nearly straight.

Mandibles, head, and body smooth and shining. Dorsa of head and body with sparse erect to suberect hairs and dense decumbent pubescence. Scapes and tibiae with abundant subdecumbent short hairs and dense decumbent pubescence. Mouthparts with abundant longer hairs, apex of mandible with a long stout hair on the ventral face. Color reddish brown. Mandibles, antennae, and legs brownish yellow.

Paratype worker: TL 2.7, HL 0.51, HW 0.40, CI 78, SL 0.40, SI 100, ML 0.25, PW 0.33, AL 0.83, PNL 0.18, PNH 0.31, PNW 0.23, PPNL 0.20, PPNH 0.30, PPNW 0.23 (1 individual measured.). As holotype.

Holotype: worker, CHINA: Tibet, Medog County, Damu Town, Damu Village, $1200 \mathrm{~m}$, collected from a soil sample in the valley tropical rain forest, 2011.VII.20, leg. Xia Liu, No.A11-3925.

Paratype: 1 worker, with the same data as holotype, but collected from a ground sample, No.A11-3863.

Comparison: This new species is close to P. rafflesi Taylor, but in profile view, posterodorsal corner of propodeum evenly convex; petiolar node strongly narrowed upward, anterior face nearly straight, dorsal face weakly convex, anterodorsal corner blunt.

Etymology: The new species is named after the type locality "Tibet".

\section{Protanilla rafflesi Taylor}

(Figs. 21-24)

Holotype worker: TL 2.7, HL 0.52, HW 0.40, CI 77, SL 0.43, SI 108, PW 0.36, AL 0.80 (Bolton, 1990); ML 0.26, PNL 0.17, PNH 0.29, PNW 0.24, PPNL 0.18, PPNH 0.33, PPNW 0.25.

In full face view, head longer than broad, anterior $1 / 3$ of the head distinctly narrowed forward. Lateral sides weakly convex, anterolateral corners 
prominent and tooth-like. Occipital margin weakly concave, occipital corners roundly prominent. Mandibles elongate and down-curved apically, dorsolateral surface with a longitudinal groove, basal corners rounded, masticatory margin with about 14 peg-like teeth. Labrum with a pair of peg-like teeth. Clypeus roughly trapezoidal, anterior margin slightly concave. Antennae 12-segmented, scapes surpassed occipital corners by about $1 / 10$ of its length, flagella segments 2-8 about as broad as long.

In profile view, pronotum slightly convex. Promesonotal suture complete but not depressed. Dorsum of mesonotum straight, almost horizontal. Metanotal groove widely shallowly notched. Dorsum of propodeum straight, slightly slope down backward; posterodorsal corner roundly strongly convex; declivity weakly convex, about $1 / 2$ length of the dorsum. Propodeal spiracle small and circular, lower down on side. Metapleural bulla elongate elliptical and horizontal, close to the spiracle. Petiolar node roughly trapezoidal, weakly narrowed upward; anterior face weakly concave, dorsal face strongly convex, posterior face nearly straight; anterodorsal corner roundly protruding, higher

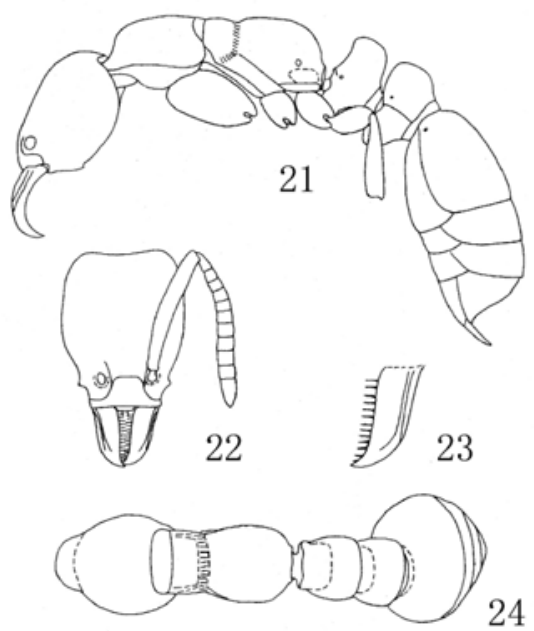

Figs. 21-24: Worker of Protanilla rafflesi Taylor; 21. Head and body in profile view; 22 . Head in full face view; 23. Mandible in dorsal view; 24. Body in dorsal view. (Drawn from images of the AntWeb, California Academy of Sciences. Pilosity omitted). than posterodorsal corner, the latter rounded; the shapes of ventral face and subpetiolar process are not clear because concealed by the hind coxa and femur. Postpetiole moderately inclined forward, dorsal face roundly convex, anterior face weakly convex, anterodorsal corner roundly extruding; anteroventral corner roundly protruding, posteroventral face weakly convex. Sting strong and extruding.

In dorsal view, petiolar node roughly rectangular and slightly widenedbackward, distinctlybroader than long, lateral sides weaklyconvex. Postpetiolar node weakly widened backward, slightly wider than petiolar node, lateral sides weakly convex. 
Mandibles, head, and body smooth and shining. Dorsa of head and body with sparse suberect hairs and abundant decumbent pubescence. Scapes with sparse subdecumbent hairs and abundant decumbent pubescence. Tibiae with abundant decumbent pubescence, but without standing hairs. Mouthparts with abundant longer hairs, apex of mandible with a long stout hair. Color brownish yellow. Mandibles, antennae, and legs yellowish.

Holotype: worker, SINGAPORE: McRitchie, 1970.IX.1, D. H. Murphy leg., deposited in the Natural History Museum, London (Bolton, 1990).

Distribution: Singapore and East Malaysia (Bolton, 1990).

Discussion: Bolton (1990) assigned P. rafflesi Taylor as the type-species for the establishment of the genus Protanilla Taylor. However, we sometimes encounter troubles when comparing an unknown species with $P$. rafflesi Taylor because no complete description of the type-species has been published since 1990. Recently, high quality images of the holotype specimen of $P$. rafflesi Taylor were made available on the AntWeb of California Academy of Sciences. In order to facilitate the comparison, a complete description of $P$. raffles $i$ Taylor is made based on the AntWeb images of the holotype specimen so as to consummate its taxonomic information. Unfortunately, the lower portion of the petiole is concealed by the hind coxa and femur in the profile image, and the shapes of the ventral face and the subpetiolar process are kept unknown.

\section{ACKNOWLEDGMENTS}

This study is supported by the National Natural Science Foundation of China (No. 30870333) and the Key Subject of Forest Protection of Yunnan Province. I thank Miss Xia Liu (Doctorate Candidate of Forest Protection, Beijing Forestry University, Beijing) and Mr. Hai-Bin Li (Student of Forest Protection, Southwest Forestry University, Kunming) for collecting the type specimens with me. I also thank California Academy of Sciences (USA) for the permission to use the images of Protanilla rafflesi Taylor from AntWeb.

\section{REFERENCES}

Baroni Urbani, C. \& De Andrade, M.L. 2006. A new Protanilla Taylor, 1990 from Sri Lanka. Myrmecologische Nachrichten 8: 45-47. 
Bolton, B. 1987. A review of the Solenopsis genus-group and revision of Afrotropical Monomorium Mayr. Bulletin of the British Museum (Natural History) (Entomology) 54: 263-452.

Bolton, B. 1990. The higher classification of the ant subfamily Leptanillinae. Systematic Entomology 15: 267-282.

Bolton, B. 1994. Identification Guide to the Ant Genera of the World. Harvard University Press, 222 pp. Cambridge, Massachusetts.

Bolton, B. 1995. A New General Catalogue of the Ants of the World. Harvard University Press, 504 pp. Cambridge, Massachusetts.

Bolton, B. 2011. An Online Catalog of the Ants of the World. http://www.antcat.org/.

Borowiec, M. L., Schulz, A, Alpert, G. D. \& Banar, P. 2011. Discovery of the worker caste and descriptions of two new species of Anomalomyrma (Hymenoptera: Formicidae: Leptanillinae) with unique abdominal morphology. Zootaxa 2814: 1-14.

Emery, C. 1870. Studi mirmecologici. Bullettino della Società Entomologica Italiana 2: 193-201.

Terayama, M. 2009. A synopsis of the family Formicidae of Taiwan. Research Bulletin of Kanto Gakuen University 17: 81-266.

$\mathrm{Xu}, \mathrm{Z}$. 2002. A systematic study on the ant subfamily Leptanillinae of China. Acta Entomologica Sinica 45: 115-120.

Xu, Z. \& Zhang, J. 2002. Two new species of the ant subfamily Leptanillinae from Yunnan, China. Acta Zootaxonomica Sinica 27: 139-144.

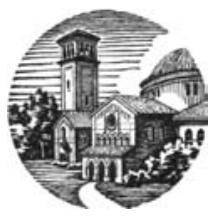


